\title{
Association of Socioeconomic Status with Medication Persistence and Adherence After an Acute Ischemic Stroke
}

Jacqueline $\mathrm{Xu}^{1}$, Janet Prvu Bettger2,3, Yuesong Pan ${ }^{4,5}$, Liping Liư 4,5 , Li Zixiao4,5, Yilong Wang ${ }^{4,5}$, Yongjun Wang ${ }^{4,5}$

${ }^{1}$ Duke University Sanford School of Public Policy, Durham, NC, USA; 2Duke University School of Medicine, Durham, NC, USA; ${ }^{3}$ Duke Global Health Institute, Duke University, Durham, NC, USA, ${ }^{4}$ Department of Neurology, Beijing Tiantan Hospital, Capital Medical University, Beijing, China; ${ }^{5}$ China National Clinical Research Center for Neurological Diseases (NCRC-ND)

\section{Background}

- Stroke is the leading cause of disease burden in China. ${ }^{1}$

- Although several medications can modify risk for stroke recurrence, few studies have examined barriers to continuing prescribed medications. . $^{2,3,4}$

- Purpose: This study examined the association of family income, health insurance and educational attainment with the continuation of prevention medications for three months following acute ischemic stroke (AIS) hospital discharge in China.

\section{Methods}

- Patients with AIS discharged from the 219 hospitals participating in the Chinese National Stroke Registry (CNSR) II

- Self-report of medications by phone 3 months from hospital discharge

- Persistence $=$ continuation of all medications prescribed at hospital discharge

- Adherence $=$ percentage of prescribed medication classes continued (per patient), compared with the study population median adherence $(67 \%)$

\section{Results}

- Among 11,868 patients, $46.8 \%$ were persistent and $57.1 \%$ were adherent with medications 3 months after hospital discharge.

- Patients with less formal education had lower adherence $(p<0.001)$ and were less persistent overall (and with antiplatelets, antihypertensives and statins) than patients with $\geq$ high school education.

- Patients with family income $\leq 1000$ $\mathrm{RMB} / \mathrm{mo}$ had lower adherence $(p<0.001)$ and were less persistent overall (and with antiplatelets and antihypertensives) than those with higher family income.

- Having health insurance was not associated with persistence or adherence.
Persistence Overall and by Medication for each Socioeconomic Status Factor

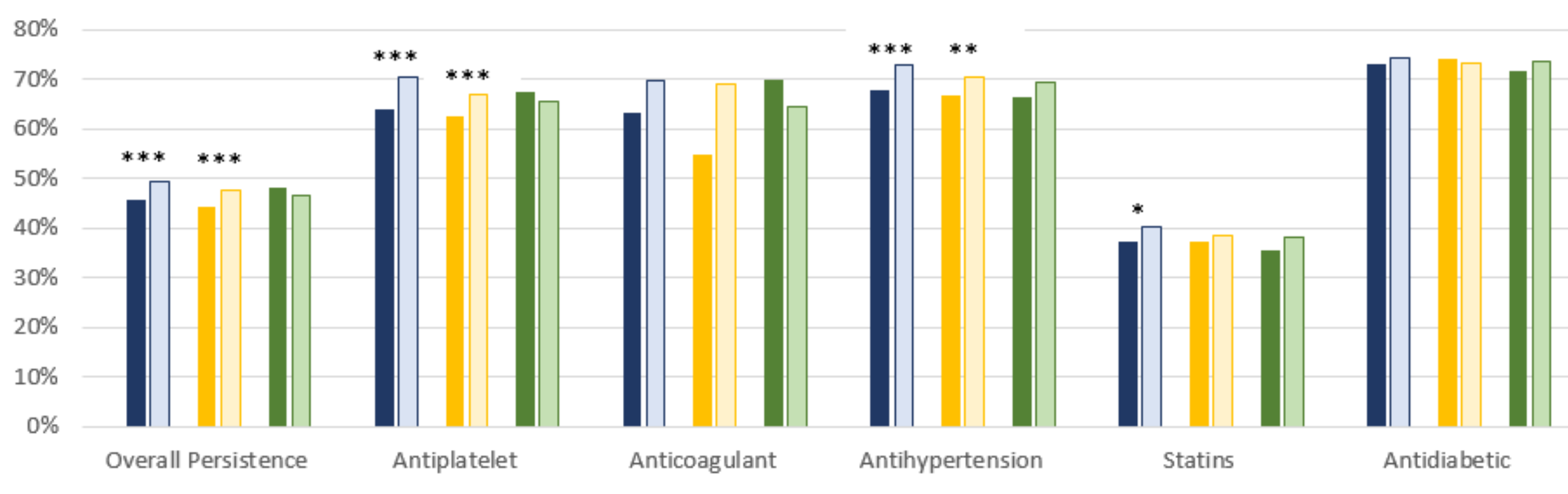

\begin{tabular}{|c|c|c|c|c|c|c|}
\hline \multirow[b]{2}{*}{$\begin{array}{l}\text { Socioeconomic } \\
\text { Status (SES) } \\
\text { Factors }\end{array}$} & \multicolumn{3}{|c|}{ PERSISTENCE } & \multicolumn{3}{|c|}{ ADHERENCE $^{\dagger}$} \\
\hline & $\begin{array}{l}\text { Unadjusted } \\
\text { OR }(95 \% \mathrm{Cl})\end{array}$ & $\begin{array}{c}\text { Adjusted for } \\
\text { patient factors } \ddagger \\
\text { OR }(95 \% \mathrm{Cl})\end{array}$ & $\begin{array}{l}\text { Adjusted for SES, } \\
\text { patient and } \\
\text { hospital factors } \S \\
\text { OR }(95 \% \mathrm{Cl})\end{array}$ & $\begin{array}{l}\text { Unadjusted } \\
\text { OR }(95 \% \mathrm{Cl})\end{array}$ & $\begin{array}{l}\text { Adjusted for patient } \\
\text { factors } \neq \text { OR }(95 \% \\
\text { CI) }\end{array}$ & $\begin{array}{c}\text { Adjusted for SES, } \\
\text { patient and hospital } \\
\text { factors } \S \\
\text { OR }(95 \% \mathrm{Cl}) \\
\end{array}$ \\
\hline $\begin{array}{l}\text { Educational } \\
\text { Attainment }\end{array}$ & \multicolumn{3}{|c|}{ (reference group: $\leq$ elementary) } & \multicolumn{3}{|c|}{ (reference group: $\leq$ elementary) } \\
\hline Middle School & $1.04(0.95-1.13)$ & $0.98(0.90-1.07)$ & $0.95(0.87-1.05)$ & $1.10(1.01-1.21)$ & $1.06(0.97-1.16)$ & $0.99(0.90-1.09)$ \\
\hline$\geq$ High School & $1.16(1.07-1.27)$ & $1.09(0.99-1.19)$ & $1.06(0.96-1.18)$ & $1.31(1.20-1.43)$ & $1.25(1.14-1.37)$ & $1.12(1.01-1.24)$ \\
\hline Family Income* & \multicolumn{3}{|c|}{$\begin{array}{c}\text { (reference group: } \leq 1000 \mathrm{RMB} / \mathrm{mo} \\
=123.49 € \text { or } \$ 160.52 \text { USD) }\end{array}$} & \multicolumn{3}{|c|}{$\begin{array}{l}\text { (reference group: } \leq 1000 \mathrm{RMB} / \mathrm{mo} \\
=123.49 € \text { or } \$ 160.52 \mathrm{USD})\end{array}$} \\
\hline $1001-3000$ & $1.19(1.10-1.30)$ & $1.16(1.07-1.27)$ & $1.18(1.07-1.29)$ & $1.22(1.12-1.33)$ & $1.20(1.10-1.31)$ & $1.15(1.05-1.26)$ \\
\hline$>3001$ & $1.03(0.92-1.15)$ & $1.00(0.89-1.12)$ & $1.07(0.94-1.22)$ & $1.21(1.08-1.35)$ & $1.19(1.06-1.34)$ & $1.12(0.99-1.27)$ \\
\hline Insurance & \multicolumn{3}{|c|}{ (reference group: self-pay) } & \multicolumn{3}{|c|}{ (reference group: self-pay) } \\
\hline $\begin{array}{l}\text { Has health } \\
\text { insurance }\end{array}$ & $0.94(0.82-1.08)$ & $0.97(0.84-1.12)$ & $0.93(0.80-1.07)$ & $0.99(0.86-1.15)$ & $1.03(0.89-1.19)$ & $0.93(0.80-1.08)$ \\
\hline
\end{tabular}

\section{Conclusions}

Half of the stroke patients in this study reported discontinuing 1 or more of their prescribed secondary prevention medications within 3 months of their index stroke hospitalization. There was an association between less education and lower family income with worse medication persistence and adherence. Targets for improving secondary stroke prevention need to be identified for all patients and those with lower socioeconomic status.

\section{References}

1. Liu L, Wang D, Wong KS, Wang Y. Stroke and stroke care in China: huge burden, significant workload, and a national priority. Stroke 2011; 42: 3651-3654

2. Jiang, Y., Yang, X., Li, Z., Pan, Y., Wang, Y., Wang, Y., ... \& Wang, C. (2017). Persistence of secondary prevention medication and related factors for acute ischemic stroke and transient ischemic attack in China. Neurological research, 39(6), 492-497.

3. Ji, R., Liu, G., Shen, H., Wang, Y., Li, H., Peterson, E., \& Wang, Y. (2013). Persistence of secondary prevention medications after acute ischemic stroke or transient ischemic attack in Chinese population: data from China National Stroke Registry. Neurological research, 35(1), 2936.

4. Xu, J., Ju, Y., Wang, C., Wang, Y., Liu, L., Zhao, X., ... \& Wang, Y. (2013). Patterns and predictors of antihypertensive medication used 1 year after ischemic stroke or TIA in urban China. Patient preference and adherence, 7,71 